\title{
METHODOLOGICAL APPROACHES TO ASSESSING THE IMPACT OF INFORMATION TECHNOLOGIES ON ECONOMIC GROWTH IN THE REGIONS OF RUSSIA ${ }^{1}$
}

\author{
Elena A. Petrova \\ Volgograd State University, Volgograd, Russian Federation \\ Petr V. Bondarenko \\ Volgograd State University, Volgograd, Russian Federation \\ Alla V. Shipileva \\ Volgograd State University, Volgograd, Russian Federation
}

\begin{abstract}
In this work, the authors propose a methodological approach to study the impact of using NBICtechnologies on the economic growth of the regions of the Russian Federation. The authors show that among NBIC-technologies they are ICT that have the greatest impact on economic growth. The assessment tools are integrated empirical analysis methods. At the first stage, a cluster analysis was carried out using the k-means method according to the per-capita GRP, the level of population income and the level of ICT use, under which three groups of Russian regions were distinguished, characterized by low, medium and high dynamics of economic growth. At the second stage, a canonical correlation analysis was carried out and analytical expressions of the interconnections of economic growth indicators and a set of indicators characterizing the development and use of ICT in the regions of the Russian Federation were obtained. The study proves the relationship between ICT and economic growth in the regions of the Russian Federation. The greatest influence is exerted by indicators such as the number of mobile cell phones and broadband Internet subscribers. The canonical correlation analysis for the selected groups of regions did not give positive results, the results for the first cluster, which is characterized by low dynamics of economic growth, turned out to be statistically significant. Most likely, this is due to the fact that in this group using ICT gives the greatest effect. However, the substantiation of this hypothesis requires the expansion of the statistical base of the study, both in time and in terms of expanding the composition of indicators that reflect not only economic, but also social aspects of the processes under study.

Key words: ICT, economic growth, NBIC-technologies, cluster analysis, canonical analysis.

Citation. Petrova E.A., Bondarenko P.V., Shipileva A.V. Methodological Approaches to Assessing the Impact of Information Technologies on Economic Growth in the Regions of Russia. Journal of Volgograd State University. Economics, 2020, vol. 22, no. 1, pp. 23-34. (in Russian). DOI: https://doi.org/10.15688/ek.jvolsu.2020.1.3

\section{МЕТОДИЧЕСКИЕ ПОДХОДЫ К ОЦЕНКЕ ВЛИЯНИЯ ИНФОРМАЦИОННЫХ ТЕХНОЛОГИЙ НА ЭКОНОМИЧЕСКИЙ РОСТ В РЕГИОНАХ РОССИИ ${ }^{1}$}

\section{Елена Александровна Петрова}

Волгоградский государственный университет, г. Волгоград, Российская Федерация

Петр Владимирович Бондаренко

Волгоградский государственный университет, г. Волгоград, Российская Федерация 


\title{
Алла Владимировна Шипилева
}

Волгоградский государственный университет, г. Волгоград, Российская Федерация

\begin{abstract}
Аннотация. В настоящей работе авторами предлагается методологический подход для исследования влияния использования информационно-коммуникационных технологий (далее - ИКТ) на экономический рост регионов РФ. Авторами показано, что среди нано-, био-, инфо- и когнитивных (далее - НБИК) технологий именно ИКТ оказывают наибольшее влияние на экономический рост. Инструментарий оценки составляют методы комплексного эмпирического анализа. На первом этапе проведен кластерный анализ методом $k$ средних по среднедушевому валому региональному продукту (далее - ВРП), уровню доходов населения и уровню использования ИКТ, посредством которого выделены три группы регионов РФ, характеризующиеся низкой, средней и высокой динамикой экономического роста. На втором этапе проведен канонический корреляционный анализ и получены аналитические выражения взаимосвязей показателей экономического роста и набора индикаторов, характеризующих развитие и использование ИКТ в регионах РФ. В исследовании доказана взаимосвязь ИКТ и экономического роста регионов РФ. Наибольшее влияние оказывают индикаторы с такими параметрами, как число мобильных сотовых телефонов и абонентов широкополосного доступа в Интернет. Канонический корреляционный анализ по выделенным группам регионов не дал положительных результатов, статистически значимыми оказались результаты для первого кластера, который характеризуется низкой динамикой экономического роста. Вероятнее всего, это связано с тем, что в этой группе использование ИКТ дает наибольший эффект. При этом обоснование данной гипотезы требует расширения статистической базы исследования как во временном, так и плане расширения состава индикаторов, отражающих не только экономические, но и социальные аспекты исследуемых процессов.
\end{abstract}

Ключевые слова: ИКТ, экономический рост, НБИК-технологии, кластерный анализ, канонический анализ.

Цитирование. Петрова Е. А., Бондаренко П. В., Шипилева А. В. Методические подходы к оценке влияния информационных технологий на экономический рост в регионах России // Вестник Волгоградского государственного университета. Экономика. - 2020. - Т. 22, № 1. - C. 23-34. - DOI: https://doi.org/10.15688/ ek.jvolsu.2020.1.3

\section{Введение}

Одной из фундаментальных проблем, обсуждаемой как в российских, так и в зарубежных исследованиях, является обоснование механизмов достижения стабильных темпов экономического роста, а также обеспечения его нового уровня качества. Основными условиями, обеспечивающими такое развитие экономики, являются технологические сдвиги, вызванные научно-техническим прогрессом, современный этап которого связан с развитием конвергентных технологий, представляющих взаимовлияние и взаимопроникновение групп НБИК-технологий, составляющих ядро нового технологического уклада и определяющих структурно-динамические процессы в хозяйственных системах. В связи с этим развитие информационного сектора экономики региона, стимулирование научных исследований в области нано-, био- и инфокоммуникационных технологий и внедрение инновационных результатов исследования в реальный сектор экономики позволит осуществить интенсификацию инновационных процессов в регионе и выйти на новый уровень экономического роста.

Инвестиции в цифровые технологии больше не ограничиваются элитной группой «высокотехнологичных» экономик, сегодня они вкладываются в развитые и развивающиеся экономики и приносят прибыль. Перед правительствами как стран, так и отдельных регионов стоит задача понять, каким образом максимально увеличить эффекты использования НБИК-технологий во всех сферах экономики и общества [Иншаков и др., 2017]. При этом все еще отсутствуют инструменты, которые позволили бы адекватно измерить и спланировать получаемые эффекты. Федеральная служба государственной статистики (Росстат) аккумулирует множество официальных статистических данных и принятых показателей, однако ни один из них в полной мере не отражает то, как цифровые технологии влияют на предприятия, отрасли и экономику в целом. В зрелых экономиках, давно идущих по пути цифровизации, НБИК-технологии проникают во все отрасли (от сельского хозяйства до высокотехнологичных производств), 
встроены во все бизнес-процессы предприятия и в результате способствуют увеличению производительности труда, а следовательно и экономическому росту [Inshakov et al., 2017].

Согласно исследованиям зарубежных и российских экспертов по проблемам развития цифровой экономики [Конкуренция в цифровую эпоху ...] существует несколько фундаментальных факторов, влияющих на рост экономики и использование НБИК-технологий:

- укрепление нецифровых основ экономики, обеспечение гибкой и благоприятной нормативной среды и оказание поддержки учреждениям, организациям и населению в процессе адаптации к цифровому миру;

- укрепление цифровых основ экономики, то есть создание масштабируемой, умной и безопасной инфраструктуры, способной адекватно реагировать на ожидаемый взрывной рост цифровой экономики;

- усиление взаимодействия, интеграции и гармонизации функционирования цифровой экосистемы (как по горизонтали - между отраслями и секторами экономики, так и по вертикали - на всех уровнях государственного управления) для поддержки инноваций и обеспечения возможности осуществления технологического прорыва, к которому стремится российское руководство;

- развитие цифровых навыков для роста процветающей цифровой экономики и появления высококвалифицированной рабочей силы;

- взаимозависимость между цифровым развитием, внедрением культуры открытых инноваций, изменением традиционных структур управления и соответствующих перемен в обществе.

Традиционно оценку экономического роста связывают с увеличением такого макроэкономического показателя, как валовой внутренний продукт (далее - ВВП), а на региональном уровне - с его аналогом - ВРП. По этой причине основу экономического роста и повышения уровня жизни населения закладывает рост производительности труда, который напрямую связан с использованием новейших технологий как в производственных, так и в управленческих процессах. Согласно последним зарубежным исследованиям именно информационные технологии в большей степени влияют на экономический рост, поэтому авторами в предлагаемом исследовании решается задача измерения эффектов использования ИКТ.

\section{Материалы и результаты исследования}

Вопрос о влиянии НБИК-технологий на производительность труда, оценка силы и поиск аналитического выражения взаимосвязи между внедрением этих технологий и устойчивым экономическим развитием широко изучалась в развитых странах на уровне фирм, отраслей и стран, причем большинство исследований показали, что влияние ИКТ на производительность является положительным и экономически значимым [Cardona et al., 2013].

Большинство исследований, посвященных макроэкономическому анализу связи между ИКТ и ростом экономики, в большей степени основаны на оценке факторов роста. В 1990-х и 2000-х гг., после опубликования «Европейской статистической палатой» промышленных данных по ИКТ, научные публикации Jorgenson, Stiroh, Oliner, Sichel были сфокусированы на результате производительности ИКТ в США [Jorgenson et al., 2000; Oliner et al. 2000; Stiroh, 2002]. Inklaar, O’Mahony, и Timmer, сравнивая вклад ИКТ в экономики США и четырех европейских стран (Франции, Германии, Нидерландов и Великобритании), пришли к выводу, что в США вклад ИКТ в развитие экономики выше, чем в вышеперечисленных европейских странах на период с 1979 по 2000 год [Inklaar et al., 2005]. С появлением базы данных EU KLEMS [O'Mahony et al., 2009] появились сравнительные исследования по европейским странам [Inklaar et al., 2008; van Ark et al., 2008; Strauss et al., 2011; Productivity ..., 2011], которые показали значительную отраслевую и международную неоднородность в отношении вклада ИКТ в рост производительности труда в развитых странах.

При этом исследования последних десятилетий по обновленным расширенным данным, которые учитывают отраслевую дифференциацию, доказывают положительный вклад ИКТ в рост производства как в Великобритании, так и в США [O'Mahony et al., 2005; Dimelis et al., 2011].

Другое направление исследований сосредоточено исключительно на коммуникативных 
технологиях. Так, Roller и Waverman доказывают наличие причинно-следственной связи между проводной электросвязью и ВВП для 21 страны ОЭСР [Roller et al., 2001]. Czernich, Falck, Kretschmer и Woessmann поддерживают выводы Roller and Waverman о важности коммуникационных технологий [Broadband Infrastructure ..., 2011]. Основываясь на группе из 20 стран ОЭСР, они предоставляют эмпирические доказательства того, что увеличение уровня проникновения широкополосной связи ведет к увеличению темпов роста ВВП. Наиболее подробные эмпирические исследования по ИКТ по развитым странам можно найти в работах других исследователей [Biagi, 2013; Cardona et al., 2013; Draca et al., 2007; The Economic Impact ...]. Кроме того, Indjikian and Siegel дают обзор количественных и качественных исследований взаимосвязи между ИКТ и экономическими показателями как в развитых, так и в развивающихся странах [Indjikian et al., 2005].

Изначально не совсем понятно, является ли вклад ИКТ в рост экономики развивающихся стран больше, чем в развитых странах. Так, Steinmueller отмечает, что «ИКТ имеют потенциал для поддержки развития стратегии "опережения", то есть в обход некоторых процессов наращивания человеческого фактора и фиксированных инвестиций для того, чтобы расширить разность в производительности труда и ВВП, которая разделяет развитые и развивающиеся страны [Steinmueller, 2001]. Если «опережение» гипотетически произойдет, то адаптационная способность ИКТ в развивающихся странах будет значительно выше, чем в развитых. При этом успех этой стратегии значительно зависит от способности и усилий работников и менеджеров применять новые технологии.

В докладе ООН [United Nations ..., 2011, c. 71-78] подробно анализируются причины, объясняющие, почему вклад ИКТ в развивающихся странах и странах с переходной экономикой может иметь более сильное влияние на экономическое развитие. Во-первых, инвестиции в ИКТ могут уменьшить административную нагрузку на фирмы путем внедрения технологий электронного правительства. Более того, ИКТ могут быть использованы для подготовки специалистов и консалтинговых услуг. Это также упростит доступ к необходимой информации, что сократит время на поездки и уменьшит операционные издержки, мобильные банковские приложения будут особенно полезны для небольших и маленьких предприятий. Ни один из вышеперечисленных сервисов ИКТ не является специфичным для развивающихся стран. При этом в указанных странах ИКТ часто обеспечивают сервисами, которые до этого не были доступны как в цифровых, так и в нецифровых экономиках.

В своем исследовании Yousefi, используя данные Всемирного Банка на период с 2000 по 2006 год, выявил незначительный вклад инвестиций в ИКТ в рост производства в развивающихся странах [Yousefi, 2011]. Paбота Dedrick, Kraemer, и Shih представляет самые новые и обширные данные по изучаемым вопросам на настоящий момент [Dedrick et al., 2013]. В статье представлены результаты по 45 развитым и развивающимся странам с высоким уровнем доходности в период с 1994 по 2007 год. Авторы предоставляют эконометрические доказательства вклада ИКТ в рост экономики как развивающихся, так и развитых стран с немного большим коэффициентом вклада инвестиций в ИКТ в развитых странах, чем в «высокодоходных развивающихся странах». Такие различия в результатах исследований могут быть объяснены использованием различных аналитических методов и баз данных по различным странам в различные несовпадающие периоды.

Незначительная производительность ИКТ, особенно в развивающихся странах, может быть обусловлена недостатком потенциала освоения или тем фактом, что получение доходов от использования ИКТ в этих странах займет какое-то время. Дополнительный рост производительности с их участием может быть обусловлен действием сетевых эффектов. Если придерживаться гипотезы «опережения», то вклад ИКТ в рост экономики развивающихся стран должен быть значительно выше, чем в экономику развитых стран. Подтверждают эти выводы и исследования Paunov и Rollo, которые, основываясь на данных Всемирного Банка по исследованию предприятий для 117 развивающихся стран, показали, что существует четкий положительный эффект на производительность 
труда от использования интернета на предприятиях [Paunov et al., 2016]. Недавнее исследование Cirera, Lage и Sabetti показало положительный эффект от применения ИКТ-инноваций, но неоднозначный результат в связи между инновациями и производительностью на примере 6 Африканских стран [Cirera et al., 2016].

На уровне регионов Российской Федерации такие исследования практически отсутствуют, что не позволяет сделать однозначного вывода о вкладе ИКТ в экономику региона. Дифференциация субъектов РФ по уровню социально-экономического развития также требует разработки методического инструментария для оценки такого влияния в каждой группе регионов, что позволит разработать программы развития, специфические для каждой группы. Несмотря на довольно неоднозначные исследования, Всемирный банк придерживается оптимистического взгляда на то, что ИКТ «...имеют большие перспективы для сокращения бедности, повышения производительности, ускорения экономического роста...» [Всемирный банк ... , 2012]. Отсутствие эмпирических исследований о влиянии ИКТ по отдельным территориям может быть в значительной степени обусловлено отсутствием адекватных данных микро- и мезоуровня по информационному сектору.

Можно выделить следующие причины, по которым влияние ИКТ на рост в регионах РФ отличается от такового в целом по стране, а также от данных, характерных для развитых стран. С одной стороны, регионам, показывающим очень низкие темпы роста среднедушевого ВРП и являющимся дотационными, не хватает внутренних ресурсов (отсутствие соответствующего уровня человеческого капитала, а также низкие расходы на НИОКР) для использования ИКТ в экономике. С другой стороны, ИКТ могли бы позволить таким территориям «перепрыгнуть» традиционные методы повышения производительности труда [Steinmueller, 2001].

Кроме того, повышение производительности труда может быть вызвано «вторичными эффектами, связанными с ИКТ, или сетевыми эффектами» [Stiroh, 2002], поскольку ИКТ могут снизить операционные издержки и ускорить процесс создания знаний [Pilat,
2004]. Эти сетевые эффекты могут быть более выраженными, «когда многие компании в регионе или отрасли используют аналогичные уровни или типы ИКТ» [Draca et al., 2007].

В предлагаемом исследовании решается задача измерения эффектов использования ИКТ, разрабатывается подход, использующий расширенный состав индикаторов и позволяющий определить наличие взаимосвязи между ИКТ и экономическим ростом.

Методический инструментарий включает в себя на первом этапе типологизацию регионов РФ по среднедушевому ВРП, уровню доходов населения и уровню использования ИКТ, на втором этапе с применением методов канонической корреляции доказывается наличие взаимосвязи, а также получены аналитические выражения этого взаимодействия для каждой группы регионов.

Эмпирический анализ, проведенный авторами в целом по всем субъектам РФ без выделения отдельных групп по уровню экономического развития, позволил сделать однозначные выводы о наличии взаимосвязи между НБИК-технологиями и экономическими ростом. Построенные эконометрические модели показали устойчивую статистическую значимость, однако дополнительные исследования по группам регионов (в ходе кластерного анализа выделено 3 группы - с высокой динамикой, со средней динамикой и с низкой динамикой экономического роста) не позволили сделать однозначные выводы о наличии такого влияния внутри группы.

Эмпирическую базу исследования составила выборка, состоящая из 410 наблюдений по соответствующим отобранным показателям и субъектам РФ за 2012-2016 годы. Массив данных был отобран с учетом корреляционного анализа с использованием параметрического метода расчета коэффициентов Браве-Пирсона $(r>0,5)$. Для проведения канонического анализа была произведена нормировка значений показателей по формуле стандартизованных значений.

В качестве показателей, отражающих экономический рост регионов РФ, были выделены следующие индикаторы: $Y_{1}-$ коэффициент демографической нагрузки, $Y_{2}-$ коэффициент естественного прироста на 10000 человек населения, $Y_{3}$ - среднедушевые денеж- 
ные доходы населения, $Y_{4}$ - валовой региональный продукт на душу населения. Характеристика экономического роста обычно ограничивается среднедушевым значением валового регионального продукта. При этом в последнее время в публикациях усиливается критика этого показателя [Гранберг и др., 2003; Зайцева, 2009; Петрова и др., 2016], к недостаткам которого относят запаздывание на 2 года его расчета, отсутствие последнего на уровне муниципальных образований и крупных городов, а также крайне ограниченную информацию об отраслевой структуре и о вкладе отдельных видов экономической деятельности в итоговое значение. Все это затрудняет выполнение анализа влияния отдельных факторов на экономический рост, особенно это касается вклада информационного сектора экономики, и практически невозможно провести адекватные оценки по вкладу нано- и биотехнологий. В связи с этим в данном исследовании предлагается расширить набор показателей, характеризующих экономический рост. Из-за отсутствия официальных данных по всем конвергентным технологиям (особенно это касается нано- и биотехнологий) авто- ры ограничились набором индикаторов, описывающих состояние и использование информационно-коммуникационных технологий.

Системой показателей, характеризующих информационный сектор экономики, вып̃тупают: $X_{21}$ - число абонентов фиксированного широкополосного доступа в Интернет на 100 чел. населения; $X_{22}$ - число абонентов мобильного широкополосного доступа в Интернет на 100 чел. населения; $X_{23}$ - число мобильных сотовых телефонов на 100 домохозяйств, шт.; $X_{24}-$ инвестиции в основной капитал по ВЭД (деятельность в области информатизации и связи); $X_{25}$ - доля домохозяйств, имеющих доступ в Интернет, \%.

На первом этапе эмпирического анализа проведен кластерный анализ методом $k$-средних, итеративный алгоритм которого минимизирует дисперсию внутри каждого кластера. Первоначально было сформировано 4 кластеpa, но последняя группа регионов была малочисленной, что не позволило провести дальнейшие исследования. В связи с этим 3-й и 4-й кластер были объединены, так как их характеристики наиболее близки. В таблице 1 представлены результаты кластерного анализа.

\section{Группы регионов Российской Федерации динамике показателей экономического роста за 2012-2016 годы}

\begin{tabular}{|c|c|}
\hline $\begin{array}{l}\text { Низкая } \\
\text { динамика } \\
\text { экономическо } \\
\text { го роста } \\
\text { (кластер 1) }\end{array}$ & $\begin{array}{l}\text { Республика Дагестан, Республика Ингушетия, Кабардино-Балкарская, Республика, } \\
\text { Чеченская Республика, Республика Тыва, Республика Адыгея, Республика Калмыкия, } \\
\text { Астраханская область, Волгоградская область, Карачаево-Черкесская Республика, } \\
\text { Республика Северная Осетия - Алания, Ставропольский край, Республика } \\
\text { Башкортостан, Республика Марий Эл, Удмуртская Республика, Чувашская } \\
\text { Республика, Оренбургская область, Республика Алтай, Республика Бурятия, } \\
\text { Республика Хакасия, Забайкальский край, Красноярский край, Омская область, } \\
\text { Томская область, Амурская область, Еврейская автономная область }\end{array}$ \\
\hline $\begin{array}{c}\text { Средняя } \\
\text { динамика } \\
\text { экономическо } \\
\text { го роста } \\
\text { (кластер 2) }\end{array}$ & $\begin{array}{l}\text { Белгородская область, Брянская область, Владимирская область, Воронежская область, } \\
\text { Ивановская область, Калужская область, Костромская область, Курская область, } \\
\text { Липецкая область, Орловская область, Рязанская область, Смоленская область, } \\
\text { Тамбовская область, Тверская область, Тульская область, Ярославская область, } \\
\text { Республика Карелия, Архангельская область, Вологодская область, Новгородская } \\
\text { область, Псковская область, Ростовская область, Республика Мордовия, Пермский } \\
\text { край, Кировская область, Нижегородская область, Пензенская область, Саратовская } \\
\text { область, Ульяновская область, Курганская область, Свердловская область, } \\
\text { Челябинская область, Алтайский край, Кемеровская область, Новосибирская область }\end{array}$ \\
\hline $\begin{array}{l}\text { Высокая } \\
\text { динамика } \\
\text { экономическо } \\
\text { го роста } \\
\text { (кластер 3) } \\
\end{array}$ & $\begin{array}{l}\text { Московская область, г. Москва, Республика Коми, Калининградская область, } \\
\text { Ленинградская область, Мурманская область, г. Санкт-Петербург, Краснодарский } \\
\text { край, Республика Татарстан, Самарская область, Тюменская область, Иркутская } \\
\text { область, Республика Саха (Якутия), Камчатский край, Приморский край, Хабаровский } \\
\text { край, Магаданская область, Сахалинская область, Чукотский автономный округ }\end{array}$ \\
\hline
\end{tabular}

Примечание. Составлено авторами. 
Дифференциация регионов по кластерам предоставит возможность сформировать адекватные программы развития регионов, позволяющие добиться устойчивого роста и использующие специфические инструменты региональной политики, наиболее эффективные для каждой группы.

На втором этапе применен метод канонических корреляций и получены аналитические выражения взаимосвязей показателей экономического роста и набора индикаторов, характеризующих распространение ИКТ в регионах РФ. Результаты канонического анализа по всем регионам представлены в таблице 2.

Значения канонического коэффициента корреляции $R$ в рассматриваемой выборке велико $(R=0,808)$, уровень статистической значимости р равен 0,0000001 , что говорит о высокой степени достоверности полученных результатов. Можно утверждать, что корреляция между взвешенными суммами канонических переменных в левом (экономический рост регионов РФ) и правом (информационный сектор экономики) множестве достаточно значительная. Значение общей избыточности показывает, что (на основании всех значений канонических корней и получения значений переменных в правом множестве) можно объяснить дисперсией переменных в левом выражении.

Проведем проверку всех канонических корней на значимость, для этого рассмотрим значения статистики хи-квадрат (табл. 3). Наибольшее число корней, которое можно извлечь, соответствует минимальному числу переменных в исследуемых подмножествах, в нашем случае 4 , то есть на уровне значимости $p<0.05$ статистически значимыми являются четыре канонических корня. По полученному значению $\mathrm{R}$ наиболее значимыми оказались первые два корня для дальнейшей интерпретации, именно они дают $83 \%$ и $67 \%$ извлеченной дисперсии по правому и левому множеству соответственно.

Анализ факторных нагрузок канонических корней позволяет определить наиболее значимые корни для правого и левого множества (см. табл. 4).

Так как второй корень (левое множество) извлекает 56 \% дисперсии, а в правом множестве первому каноническому корню соответствуют наибольшие нагрузки относительно переменных, связанных информационным сектором, для построения канонических переменных оставляем в рассмотрении второй (левое множество) и первый (правое множество) корень.

Следующим этапом анализа является построение канонических переменных, которые дают аналитическое выражение взаимосвязи изучаемых переменных. Для этого необходимо рассмотреть структуру факторных нагрузок по отобранным корням по левому и

Результаты канонического анализа

Таблииа 2

\begin{tabular}{|l|l|l|}
\hline \multicolumn{1}{|c|}{ Canonical analysis } & \multicolumn{1}{c|}{ Left set } & \multicolumn{1}{c|}{ Right set } \\
\hline Number of variables & 4 & 5 \\
\hline Variance extracted & $98,000 \%$ & $92,48 \%$ \\
\hline Total redundancy & $39,6921 \%$ & 39,3386 \\
\hline Canonical R & 0.808 \\
\hline$p$ & 0.000000 \\
\hline
\end{tabular}

Примечание. Составлено авторами.

Статистика хи-квадрат для канонических корней

\begin{tabular}{|c|c|c|r|r|r|c|}
\hline \multirow{2}{*}{$\begin{array}{c}\text { Root } \\
\text { Removed }\end{array}$} & \multicolumn{6}{|c|}{ Chi-Square Tests with Successive Roots Removed } \\
\cline { 2 - 7 } & $\begin{array}{c}\text { Canonical } \\
\text { R }\end{array}$ & $\begin{array}{c}\text { Canonical } \\
\text { R-sqr. }\end{array}$ & Chi-sqr. & \multicolumn{1}{c|}{$\mathbf{d f}$} & $\mathbf{p}$ & $\begin{array}{c}\text { Lambda } \\
\text { Prime }\end{array}$ \\
\hline $\mathbf{0}$ & 0,808425 & 0,653551 & 117,0010 & 20 & 0,000000 & 0,205750 \\
\hline $\mathbf{1}$ & 0,603406 & 0,364098 & 38,5594 & 12 & 0,000125 & 0,593883 \\
\hline $\mathbf{2}$ & 0,249700 & 0,062350 & 5,0588 & 6 & 0,536304 & 0,933922 \\
\hline $\mathbf{3}$ & 0,063055 & 0,003976 & 0,2948 & 2 & 0,862949 & 0,996024 \\
\hline
\end{tabular}

Таблица 3

Примечание. Составлено авторами. 
правому множеству, которые представлены в таблицах 5 и 6.

Как видно из таблицы 5, для второго корня наибольшие значения соответствуют переменным $Y_{1}-$ коэффициент демографической нагрузки, $Y_{3}$ - среднедушевые денежные доходы населения, $Y_{4}$ - валовый региональный продукт на душу населения.

Согласно таблице 6 по 1-му корню из рассмотрения исключаем переменную $X_{22}$.

Таким образом, проведенное авторами исследование позволило доказать наличие зависимости между экономическим ростом и показателями, характеризующими использование ИКТ. Полученные факторные структуры правого и левого множества (табл. 5 и 6) доказывают, что максимальный коэффициент канонической корреляции $r_{1}=0,808$ достигается в том случае, когда исходные стандартизованные переменные образуют следующую пару канонических переменных:

$$
\begin{aligned}
& U_{1}=0,96 Y_{1}-0,77 Y_{3}-0,73 Y_{4} \\
& V_{1}=0,76 X_{21}-0,9 X_{23}+0,68 X_{24}+0,68 X_{25} .
\end{aligned}
$$

\section{Заключение}

В исследовании доказана значимость ИКТ для экономического роста регионов РФ, полученные канонические переменные характеризуют силу связи между признаками-факторами $\left(X_{21}-X_{25}\right)$ и результирующими признаками $\left(Y_{1}-Y_{4}\right)$. Наибольшее влияние на экономический рост оказывает показатель $X_{23}-$ число мобильных сотовых телефонов на 100 домохозяйств и $X_{21}$ - число абонентов фиксированного широкополосного доступа в Интернет на 100 чел. населения.

Представляет также интерес аналогичный анализ по выделенным кластерам регионов. При этом проведенный авторами аналогичный анализ по группам регионов не дал положительных результатов, статистически зна-

Доли извлеченной дисперсии левого и правого множества

\begin{tabular}{|l|c|c|}
\hline \multirow{2}{*}{ Factor } & \multicolumn{2}{|c|}{$\begin{array}{c}\text { Variance Extracted (Proportions), } \\
\text { left set }\end{array}$} \\
\cline { 2 - 3 } & $\begin{array}{c}\text { Variance } \\
\text { extracted }\end{array}$ & Reddncy. \\
\hline Root 1 & 0,279754 & 0,182834 \\
\hline Root 2 & 0,560920 & 0,204230 \\
\hline Root 3 & 0,094425 & 0,005887 \\
\hline Root 4 & 0,064901 & 0,000258 \\
\hline
\end{tabular}

\begin{tabular}{|l|c|c|}
\hline \multirow{2}{*}{ Factor } & \multicolumn{2}{|c|}{$\begin{array}{c}\text { Variance Extracted (Proportions), } \\
\text { right set }\end{array}$} \\
\cline { 2 - 3 } & $\begin{array}{c}\text { Variance } \\
\text { extracted }\end{array}$ & Reddncy. \\
\hline Root 1 & 0,466779 & 0,305064 \\
\hline Root 2 & 0,214715 & 0,078177 \\
\hline Root 3 & 0,157210 & 0,009802 \\
\hline Root 4 & 0,086266 & 0,000343 \\
\hline
\end{tabular}

Примечание. Составлено авторами.

Таблииа 5

Структура факторных нагрузок (левое множество)

\begin{tabular}{|c|c|c|c|c|}
\hline \multirow{2}{*}{ Variable } & \multicolumn{4}{|c|}{ Factor Structure, left set } \\
\cline { 2 - 5 } & Root 1 & Root 2 & Root 3 & Root 4 \\
\hline$Y_{1}$ & 0,106995 & 0,958935 & $-0,246240$ & $-0,091438$ \\
\hline$Y_{2}$ & $-0,829611$ & $-0,440438$ & $-0,269318$ & $-0,212668$ \\
\hline$Y_{3}$ & 0,471584 & $-0,768877$ & $-0,425632$ & 0,072629 \\
\hline$Y_{4}$ & 0,443759 & $-0,734144$ & $-0,251739$ & $-0,448039$ \\
\hline
\end{tabular}

Примечание. Составлено авторами.

Структура факторных нагрузок (правое множество)

Таблича 6

\begin{tabular}{|c|c|c|c|c|}
\hline \multirow{2}{*}{ Variable } & \multicolumn{4}{|c|}{ Factor Structure, right set } \\
\cline { 2 - 5 } & Root 1 & Root 2 & Root 3 & Root 4 \\
\hline$X_{21}$ & 0,758433 & $-0,138082$ & $-0,227297$ & 0,327442 \\
\hline$X_{22}$ & 0,143903 & $-0,831610$ & $-0,402107$ & $-0,158022$ \\
\hline$X_{23}$ & $-0,900412$ & $-0,173384$ & $-0,095258$ & 0,387452 \\
\hline$X_{24}$ & 0,680698 & $-0,444563$ & 0,560918 & 0,155648 \\
\hline$X_{25}$ & 0,681084 & $-0,367741$ & 0,498993 & 0,353266 \\
\hline
\end{tabular}

Примечание. Составлено авторами. 
чимыми оказались результаты, полученные для 1-го кластера, с наиболее низкой динамикой экономического роста. Здесь нельзя сделать однозначных выводов, но тем не менее можно предположить, что именно в этой группе использование ИКТ дает наибольший эффект. Для обоснования этого вывода требуется проведение дальнейших исследований.

Стоит упомянуть еще два вопроса. Хотя нынешний набор данных охватывает большинство регионов (80 субъектов РФ), однако по некоторым из них отсутствуют значения в официальной статистике или значения показателей пришлось исключить из рассмотрения как наиболее сильно отличающиеся от остальных (выбросы). Следовательно, может иметь место смещение выбора в сторону территорий, которые используют ИКТ более эффективно, в результате чего уменьшается возможность обобщения результатов. Кроме того, при изучении влияния ИКТ следует принимать во внимание не только экономические, но также политические и социальные аспекты, такие как более легкий доступ к информации, открытость региональных властей, но это также требует дополнительных исследований.

Авторы также не включили в исследуемые множества показатели, характеризующие статистическую информацию об использовании информационных и коммуникационных технологий в организациях практически по видам экономической деятельности. Это связано с тем, что данные аккумулируются в ходе выборочных наблюдений по ограниченному числу организаций, что также влияет на статистическую значимость получаемых результатов.

Дополнительный анализ, основанный на больших размерах выборки с точки зрения периода (например, за последние 10 лет), а также количества наблюдаемых объектов и признаков, позволил бы использовать не только статистические методы, но и методы интеллектуального анализа данных (построение нейронных сетей требует больших массивов данных, кроме того, встает вопрос об однородности полученных совокупностей). Это особенно важно в отношении потенциальных проблем эндогенности в рамках полученных оценок мезоуровня. Кроме того, дополнительные исследования на уровне отдельных пред- приятий могут помочь получить более глубокое представление о влиянии ИКТ на производительность труда (а следовательно и на экономический рост) в отдельных группах.

\section{ПРИМЕЧАНИЕ}

${ }^{1}$ Исследование выполнено при финансовой поддержке РФФИ и Администрации Волгоградской области в рамках научного проекта № 19-410340014 p_а «Совершенствование механизмов и инструментов экономического роста регионов РФ в условиях развития конвергентных технологий».

The reported study was funded by RFBR and the Volgograd Region Administration in the framework of research project no. 19-410-340014 p_a "Improvement of Mechanisms and Instruments of Economic Growth in Russian Regions in Terms of Development of Convergent Technologies".

\section{СПИСОК ЛИТЕРАТУРЫ}

Гранберг, А. Г. Валовой региональный продукт: межрегиональные сравнения и динамика : монография / А. Г. Гранберг, Ю. С. Зайцева. М. : СОПС, 2003.-116 c.

Зайцева, Ю. С. Международный опыт построения региональных счетов / Ю. С. Зайцева // Региональная экономика: теория и практика. 2009. - № 34 (127). - С. 7-18.

Иншаков, О. В. Кластерное развитие российской наноиндустрии как стратегический компонент «умной специализации» регионов / О. В. Иншаков, Е. И. Иншакова // Региональная экономика. Юг России. - 2017. - № 4 (18). - С. 4-15.

Конкуренция в цифровую эпоху: стратегические вызовы для Российской Федерации // Международный банк реконструкции и развития. Всемирный банк. - Электрон. текстовые дан. Режим доступа: https://www.vsemirnyjbank. org/ru/country/russia/publication/competing-indigital-age. - Загл. с экрана.

Петрова, Е. А. Формирование системы оценки эффективности территориального управления / Е. А. Петрова, В. В. Калинина, А. В. Шевандрин // Государственное управление. 2016. - № 54. - C. 89-105.

Biagi, F. ICT and Productivity: A Review of the Literature (JRC-1PTS Working Papers on Digital Economy no. 2013-09)/ F. Biagi. - Luxembourg : Publications Office of the European Union, 2013.-70 p.

Broadband Infrastructure and Economic Growth / N. Czernich [et al.] // The Economic Journal. 2011. - № 121 (552). - P. 505-532. 
Cardona, M. ICT and Productivity: Conclusions from the Empirical Literature / M. Cardona, T. Kretschmer, T. Strobel // Information Economics and Policy. - 2013. - № 25 (3). P. $109-125$.

Cirera, X. ICT Use, Innovation, and Productivity: Evidence from Sub-Saharan Africa / X. Cirera, F. Lage, L. Sabetti. - Electronic text data. - Mode of access: https://ssrn.com/abstract=2866599. Title from screen.

Dedrick, J. Information Technology and Productivity in Developed and Developing Countries / J. Dedrick, K. L. Kraemer, E. Shih // Journal of Management Information Systems. - 2013. - № 30 (1). - P. 97-122.

Dimelis, S. FDI and ICT Effects on Productivity Growth: A Comparative Analysis of Developing and Developed Countries / S. Dimelis, S. Papaioannou // European Journal of Development Research. - 2010. - № 22 (1). P. 79-96.

Draca, M. Productivity and ICT: A Review of the Evidence/ M. Draca, R. Sadun, J. van Reenen ; R. Mansell (ed.) // The Oxford Handbook of Information and Communication Technologies. L. : Oxford University Press, 2007. - P. 100-147.

Indjikian, R. The Impact of Investment in IT on Economic Performance: Implications for Developing Countries / R. Indjikian, D. S. Siegel // World Development. - 2005. - № 33 (5). P. 681-700.

Inklaar, R. ICT and Europe's Productivity Performance: Industry-Level Growth Account Comparisons with the United States / R. Inklaar, M. O’Mahony, M. P. Timmer // Review of Income and Wealth. 2005. - № 51 (4). - P. 505-536.

Inklaar, R. Market Services Productivity Across Europe and the Us / R. Inklaar, M. P. Timmer, B. van Ark // Economic Policy. - 2008. Vol. 23 (53). - P. 139-194.

Inshakov, O. World Market for Nanomaterials: Structure and Trends in MATEC Web of Conferences /O. Inshakov, E. Inshakova// ICMTMTE.-2017.Vol. 129. - P. 1-5.

Jorgenson, D. W. Raising the Speed Limit: U.S. Economic Growth in the Information Age / D. W. Jorgenson, K. J. Stiroh // Brookings Papers on Economic Activity. - 2000. Vol. 37 (1). - P. 125-236.

O’Mahony, M. Output, Input and Productivity Measures at the Industry Level: The EU KLEMS Database / M. O’Mahony, M. P. Timmer // Economic Journal. - 2009. - Vol. 119 (538). P. F374-F403.

O’Mahony, M. Quantifying the Impact of ICT Capital on Output Growth: A Heterogeneous Dynamic
Panel Approach / M. O’Mahony, M. Vecchi // Economica. - 2005. - Vol. 72 (288). - P. 615-633.

Oliner, S. D. The Resurgence of Growth in the Late 1990s: Is Information Technology the Story? / S. D. Oliner, D. E. Sichel // Journal of Economic Perspectives. 2000. - Vol. 14 (4). - P. 3-22.

Paunov, C. Has the Internet Fostered Inclusive Innovation in the Developing World? / C. Paunov, V. Rollo// World Development. - 2016. - № 78. P. 587-609.

Pilat, D. The ICT Productivity Paradox: Insights from Micro Data / D. Pilat // OECD Economic Studies. 2004. - №38. - P. 37-65.

Productivity and Economic Growth in Europe: A Comparative Industry Perspective/M. P. Timmer [et al.] // International Productivity Monitor. 2011. - №21.-P.3-23.

Roller, L-H. Telecommunications Infrastructure and Economic Development: A Simultaneous Approach / L-H. Roller, L. Waverman // American Economic Review.-2001.-Vol. 91 (4). -P.909-923.

Steinmueller, W. E. ICTs and the Possibilities for Leapfrogging by Developing Countries / W. E. Steinmueller // International Labour Review. - 2001. - Vol. 140 (2). - P. 193-210.

Stiroh, K. J. Are ICT Spillovers Driving the New Economy? / K. J. Stiroh // Review of Income and Wealth. - 2002. - Vol. 48 (1). - P. 33-57.

Strauss, H. ICT Capital and Productivity Growth / H. Strauss, B. Samkharadze// EIB Papers. - 2011.Vol. 16(2). - P. 8-28

The Economic Impact of ICT / J. van Reenen [et al.] // Centre for Economic Performance, London School of Economics, 2010. - Electronic text data. - Mode of access: http:/cep.lse.ac.uk/ textonly/_new/research/productivity/Manage ment/PDF/breugel_cStudyThelmpact ofICTLSE.pdf (accessed 20 November 2019).

United Nations. Information Economy Report 2011: ICTs as an Enabler for Private Sector Development. United Nations Conference on Trade and Development. United Nations Publications, 2011. - Electronic text data. - Mode of access: http:/unctad.org/en/ PublicationsLibrary/ier201 1_en.pdf (accessed 20 November 2019).

van Ark, B. The Productivity Gap Between Europe and the United States: Trends and Causes / B. van Ark, M. O'Mahony, M. P. Timmer // The Journal of Economic Perspectives. - 2008. - Vol. 22 (1). P. 25-44.

Yousefi, A. The Impact of Information and Communication Technology on Economic Growth: Evidence from Developed and Developing Countries / A. Yousefi // Economics of Innovation and New Technology. - 2011. Vol. 20 (6). - P. 581-596. 


\section{REFERENCES}

Granberg A.G., Zaytseva Yu.S. Valovoy regionalnyy produkt: mezhregionalnye sravneniya $i$ dinamika: monografiya [Gross Regional Product: Interregional Comparisons and Dynamics]. Moscow, SOPS, 2003. $116 \mathrm{p}$.

Zaytseva Yu.S. Mezhdunarodnyy opyt postroeniya regionalnykh schetov [The International Experience of Building Regional Accounts]. Regionalnaya ekonomika: teoriya i praktika [Regional Economics: Theory and Practice], 2009, no. 34 (127), pp. 7-18.

Inshakov O.V., Inshakova E.I. Klasternoe razvitie rossiyskoy nanoindustrii kak strategicheskiy komponent «umnoy spetsializatsii» regionov [Cluster Development of the Russian Nanoindustry as a Strategic Component of "Smart Specialization" of Regions]. Regionalnaya ekonomika. Yug Rossii [Regional Economy. South of Russia], 2017, no. 4 (18), pp. 4-15.

Konkurentsiya $\mathrm{v}$ tsifrovuyu epokhu: strategicheskie vyzovy dlya Rossiyskoy Federatsii [Competition in the Digital Age: Strategic Challenges for the Russian Federation]. Mezhdunarodnyy bank rekonstruktsii i razvitiya. Vsemirnyy bank [International Bank for Reconstruction and Development. World Bank]. URL: https:// www.vsemirnyjbank.org/ru/country/russia/ publication/competing-in-digital-age.

Petrova E.A., Kalinina V.V., Shevandrin A.V. Formirovanie sistemy otsenki effektivnosti territorialnogo upravleniya [Formation of a System for Assessing the Effectiveness of Territorial Management]. Gosudarstvennoe upravlenie. Elektronnyy vestnik [E-journal. Public Administration], 2016, no. 54, pp. 89-105.

Biagi, F. ICT and Productivity: A Review of the Literature (JRC-1PTS Working Papers on Digital Economy no. 2013-09). Luxembourg, Publications Office of the European Union, 2013.70 p.

Czernich N., Falck O., Kretschmer T., Woessmann L. Broadband Infrastructure and Economic Growth. The Economic Journal, 2011, no. 121 (552), pp. 505-532.

Cardona M., Kretschmer T., Strobel T. ICT and Productivity: Conclusions from the Empirical Literature. Information Economics and Policy, 2013, no. 25 (3), pp. 109-125.

Cirera X., Lage F., Sabetti L. ICT Use, Innovation, and Productivity: Evidence from Sub-Saharan Africa. URL: https://ssrn.com/abstract=2866599.

Dedrick J., Kraemer K.L., Shih E. Information Technology and Productivity in Developed and Developing Countries. Journal of Management Information Systems, 2013, no. 30 (1), pp. 97-122.
Dimelis S., Papaioannou S. FDI and ICT Effects on Productivity Growth: A Comparative Analysis of Developing and Developed Countries. European Journal of Development Research, 2010, no. 22 (1), pp. 79-96.

Draca M., Sadun R., Van Reenen J. Productivity and ICT: A Review of the Evidence. The Oxford Handbook of Information and Communication Technologies. London, Oxford University Press, 2007, pp. 100-147.

Indjikian R., Siegel D.S. The Impact of Investment in IT on Economic Performance: Implications for Developing Countries. World Development, 2005, no. 33 (5), pp. 681-700.

Inklaar R., O'Mahony M., Timmer M.P. ICT and Europe's Productivity Performance: IndustryLevel Growth Account Comparisons with the United States. Review of Income and Wealth, 2005, no. 51 (4), pp. 505-536.

Inklaar R., Timmer M.P., Van Ark B. Market Services Productivity Across Europe and the Us. Economic Policy, 2008, vol. 23 (53), pp. 139-194.

Inshakov O., Inshakova E. World Market for Nanomaterials: Structure and Trends in MATEC Web of Conferences. ICMTMTE, 2017, vol. 129, p. 1-5.

Jorgenson D.W., Stiroh K.J. Raising the Speed Limit: U.S. Economic Growth in the Information Age. Brookings Papers on Economic Activity, 2000, vol. 37 (1), pp. 125-236.

O'Mahony M., Timmer M.P. Output, Input and Productivity Measures at the Industry Level: The EU KLEMS Database. Economic Journal, 2009, vol. 119 (538), pp. F374-F403.

O'Mahony M., Vecchi M. Quantifying the Impact of ICT Capital on Output Growth: A Heterogeneous Dynamic Panel Approach. Economica, 2005, vol. 72 (288), pp. 615-633.

Oliner S.D., Sichel D.E. The Resurgence of Growth in the Late 1990s: Is Information Technology the Story? Journal of Economic Perspectives, 2000, vol. 14 (4), pp. 3-22.

Paunov C., Rollo V. Has the Internet Fostered Inclusive Innovation in the Developing World? World Development, 2016, no. 78, pp. 587-609.

Pilat D. The ICT Productivity Paradox: Insights from Micro Data. OECD Economic Studies, 2004, no. 38 , pp. 37-65.

Timmer M.P., Inklaar R., O'Mahony M., van Ark B. Productivity and Economic Growth in Europe: A Comparative Industry Perspective. International Productivity Monitor, 2011, no. 21, pp. 3-23.

Roller L-H., Waverman L. Telecommunications Infrastructure and Economic Development: A Simultaneous Approach. American Economic Review, 2001, vol. 91 (4), pp. 909-923. 
Steinmueller W.E. ICTs and the Possibilities for Leapfrogging by Developing Countries. International Labour Review, 2001, vol. 140 (2), pp. 193-210.

Stiroh K.J. Are ICT Spillovers Driving the New Economy? Review of Income and Wealth, 2002, vol. 48 (1), pp. 33-57.

Strauss H., Samkharadze B. ICT Ñapital and Productivity Growth. EIB Papers, 2011, vol. 16 (2), pp. 8-28.

Bloom N., Draca M., Kretschmer T., Sadun R. The Economic Impact of ICT. Centre for Economic Performance, London School of Economics, 2010. URL: http://cep.lse.ac.uk/textonly/_new/ research/productivity/Management/PDF/ breugel_cStudyThelmpactofICTLSE.pdf (accessed 20 November 2019).
United Nations. Information Economy Report 2011: ICTS as an Enabler for Private Sector Development. United Nations Conference on Trade and Development. United Nations Publications, 2011. URL: http://unctad.org/en/PublicationsLibrary/ ier2011_en.pdf (accessed 20 November 2019).

Ark B. van., O'Mahony M., Timmer M.P. The Productivity Gap Between Europe and the United States: Trends and Causes. The Journal of Economic Perspectives, 2008, vol. 22 (1), pp. $25-44$.

Yousefi A. The Impact of Information and Communication Technology on Economic Growth: Evidence from Developed and Developing Countries. Economics of Innovation and New Technology, 2011, vol. 20 (6), pp. 581-596.

\section{Information About the Authors}

Elena A. Petrova, Doctor of Sciences (Economics), Professor, Head of the Department of Applied Informatics and Mathematical Methods in Economics, Volgograd State University, Prosp. Universitetsky, 100, 400062 Volgograd, Russian Federation, ea_petrova@mail.ru, https://orcid.org/0000-0002-6417-9498

Petr V. Bondarenko, Candidate of Sciences (Physics and Mathematics), Associate Professor, Department of Applied Informatics and Mathematical Methods in Economics, Volgograd State University, Prosp. Universitetsky, 100, 400062 Volgograd, Russian Federation, bondarenko.volsu@gmail.com, https:// orcid.org/0000-0002-5154-9664

Alla V. Shipileva, Senior Lecturer, Department of Applied Informatics and Mathematical Methods in Economics, Volgograd State University, Prosp. Universitetsky, 100, 400062 Volgograd, Russian Federation, ashipileva@mail.ru, https://orcid.org/0000-0003-2482-4955

\section{Информация об авторах}

Елена Александровна Петрова, доктор экономических наук, профессор, заведующая кафедрой прикладной информатики и математических методов в экономике, Волгоградский государственный университет, просп. Университетский, 100, 400062 г. Волгоград, Российская Федерация, ea_petrova@mail.ru,https://orcid.org/0000-0002-6417-9498

Петр Владимирович Бондаренко, кандидат физико-математических наук, доцент кафедры прикладной информатики и математических методов в экономике, Волгоградский государственный университет, просп. Университетский, 100, 400062 г. Волгоград, Российская Федерация, bondarenko.volsu@gmail.com, https://orcid.org/0000-0002-5154-9664

Алла Владимировна Шипилева, старший преподаватель кафедры прикладной информатики и математических методов в экономике, Волгоградский государственный университет, просп. Университетский, 100, 400062 г. Волгоград, Российская Федерация, ashipileva@mail.ru, https://orcid.org/0000-0003-2482-4955 